\title{
Handlungsspielräume für Tarifparteien und Interessenvertretungen bei der Arbeitszeitgestaltung
}

\begin{abstract}
Mit Blick auf krisenbedingte Arbeitslosigkeit wird Arbeitszeitflexibilität dieser Tage häufig sehr positiv diskutiert, ebenso unter dem Blickwinkel der Bedürfnisse von Beschäftigten nach Vereinbarkeit von Beruf und Sorgeverpflichtungen. Seiner Zielsetzung nach ist das Arbeitszeitrecht jedoch zunächst dem Schutz der Gesundheit der Arbeitnehmer verpflichtet - ein Aspekt, dem auch die Tarif- und Betriebsparteien bei der Ausnutzung ihrer Gestaltungsspielräume Rechnung tragen sollten.
\end{abstract}

\section{Einleitung}

Die Finanz- und Wirtschaftskrise hat die Bedeutung der tarifvertraglichen und betrieblichen Arbeitszeitgestaltung offensichtlich gemacht: Es waren flexible Arbeitszeitsysteme, die gesunkene Produktionsauslastungen abfangen oder mildern konnten, und die Bereitschaft der Beschäftigten zur Arbeitszeit- und Einkommensreduktion, die den Unternehmen „Luft zum Atmen“ verschaffte. Für die nähere Zukunft stellen sich nun wieder andere Arbeitszeitfragen. Einerseits haben Arbeitgeber großes Interesse an flexiblen Arbeitszeitmodellen, die zuschlagsfreie Überstunden bzw. Mehrarbeit oder flexible Bereitschaftsdienste erlauben. Andererseits gibt es nicht wenige Beschäftigte, die flexible Arbeitszeiten wünschen und dabei sogar Überstunden oder Wochenenddienste dulden oder gar nachfragen. Schließlich ist daran zu erinnern, dass dem Arbeitszeitrecht der keineswegs durchgängig dispositive Gesundheitsschutz immanent ist. In dieser vielschichtigen Interessenlage räumt das Arbeitszeitgesetz (ArbZG) Tarifverträgen und Betriebsvereinbarungen große Gestaltungsmöglichkeiten ein.

\section{Einordnung des Arbeitszeitrechts}

Die vielfältigen und unterschiedlichen betrieblichen Regelungen zur Arbeitszeit verdecken oftmals den Blick darauf, welche Wurzeln das Arbeitszeitrecht eigentlich hat: Das Arbeitszeitrecht gehört zum Arbeitsschutzrecht, das staatlicherseits und auf Grundlage von Art. 2 Abs. 2 GG organisiert ist (Däubler et al. 2010, $\$ 7$ Rdnr. 1). Das Bundesverwaltungsgericht (BVerwG, 19.9.2000, 1 C 17/99, Rz. 16) formuliert: "Seine [das Arbeitszeitgesetz] Aufgabe ist es, den Arbeitnehmer vor Gefahren zu schützen, die durch Überschreiten der zeitlichen Leistungsgrenzen namentlich für die Gesundheit drohen. Zugleich soll dem Arbeitnehmer im Interesse seiner Menschenwürde und der Erhaltung seiner Persönlichkeit ausreichend Freizeit erhalten bleiben".

Das Bundesverfassungsgericht hat Ähnliches zum Arbeitszeitrecht bereits in seinem Urteil zum Verbot der Nachtarbeit für Arbeiterinnen manifestiert (BVerfG v. 28.01.1992, 1 BvL 16/83). Dieses steht aber in der betrieblichen Praxis in einem Spannungsverhältnis zwischen öffentlich-rechtlichem Arbeitsschutz, der Vereinbarkeit von Familie und Beruf sowie der Vertragsfreiheit und der gewünschten wirtschaftlichen Flexibilität der Unternehmen. Eine solche, eher ökonomische Betrachtungsweise der Arbeitszeit darf aber nicht über die beschriebene Herkunft des Arbeitszeitrechts hinwegtäuschen. Das Arbeitszeitrecht setzt daher vor allem rechtliche Grenzen für Flexibilität und Handlungsoptionen.

Bestätigt wird diese Sichtweise durch europäische Vorgaben. Im Erwägungsgrund 4 der Arbeitszeitrichtlinie 2003/88/ EG heißt es: „Die Verbesserung von Sicherheit, Arbeitshygiene und Gesundheitsschutz der Arbeitnehmer bei der Arbeit stellen Zielsetzungen dar, die keinen rein wirtschaftlichen Überlegungen untergeordnet werden dürfen".

Auch in der Rechtsprechung des Europäischen Gerichtshofs (EuGH) wird dieser Aspekt deutlich. In den bekannten Urteilen Jaeger (EuGH v. 9.9.2003, C-151/02) und zuvor SIMAP (EuGH v. 3.10.2000, C-303/98) hat das Gericht Bereitschaftsdienste als Arbeitszeit bewertet und dies auch auf Arbeitsschutzaspekte gestützt. Nicht zuletzt formuliert Art. 31 Abs. 1 der jetzt verbindlichen EU-Grundrechtecharta: „Jede Arbeitnehmerin und jeder Arbeitnehmer hat das Recht auf gesunde, sichere und würdige Arbeitsbedingungen."

Das deutsche Arbeitszeitrecht erfüllt diese Vorgaben nicht durchgängig. Anders als in der europäischen Arbeitszeitrichtlinie wurde der Gesetzeszweck im deutschen Arbeitszeitgesetz nämlich nur dahingehend bestimmt, die Sicherheit und den Gesundheitsschutz „zu gewährleisten“ ( 1 Nr. 1 HS 1 ArbZG). Damit bleibt das Gesetz bereits in seiner Zweckbestimmung hinter der EU-Arbeitszeitrichtlinie zurück,

Jens M. Schubert, Dr., ist Leiter des Bereichs Recht und Rechtspolitik in der Bundesverwaltung der Vereinten Dienstleistungsgewerkschaft (ver.di) sowie Professor für Arbeitsrecht und Europäisches Recht an der Leuphana Universität Lüneburg. Arbeitsschwerpunkte: Kollektives und europäisches Arbeitsrecht. e-mail: jens.schubert@verdi.de Kerstin Jerchel ist Volljuristin und Gewerkschaftssekretärin im Bereich Recht und Rechtspolitik der Bundesverwaltung von ver.di. Arbeitsschwerpunkte: Arbeitsschutz und europäisches Arbeitsrecht. e-mail: kerstin.jerchel@verdi.de 
deren Zielsetzung ja die Verbesserung von Sicherheit, Arbeitshygiene und Gesundheitsschutz ist.

Ein noch größerer Kontrast zeigt sich in der zweiten Zweckbestimmung des deutschen Gesetzes, mit der eine Verbesserung der Rahmenbedingungen für flexible Arbeitszeiten festgeschrieben wurde ( $\$ 1$ Nr. 1 HS 2 ArbZG). Diese Zielsetzung darf jedoch nicht zur völlig freien Arbeitszeiteinteilung durch Arbeitgeber oder Beschäftigte führen (vgl. Buschmann/Ulber 2009, § 1 Rdnr. 9). Vielmehr sind die Grenzen der werktäglichen Arbeitszeiten und der arbeitsfreien Zeiten, die die $\$ \$ 3-6$ ArbZG vorgeben, als Grundansatz zu beachten. Forderungen nach einer flexiblen Arbeitszeit außerhalb dieser Vorschriften bedürfen laut $\$ 7$ ArbZG einer Rechtsgrundlage in einem Tarifvertrag und sind immer nur dann möglich und akzeptabel, wenn eine Verbesserung von Sicherheit und Gesundheitsschutz gegeben ist. Das heißt, im Zweifel unterliegt das Flexibilisierungsinteresse dem verfassungsrechtlich verbrieften Recht der Beschäftigten auf körperliche Unversehrtheit.

\section{Grundregeln des Arbeitszeitrechts}

\subsection{ZUR LÄNGE DES ARBEITSTAGES}

In $\S 3$ ArbZG ist der Acht-Stunden-Tag verankert. Daraus ergibt sich bei einer SechsTage-Arbeitswoche (Werktage sind alle Tage, die nicht Sonn- oder Feiertage sind) rechnerisch eine wöchentliche Höchstarbeitszeit von 48 Stunden. Das deutsche Arbeitszeitrecht knüpft aber, anders als die Europäische Arbeitszeitrichtlinie (und der dortige Artikel 6 lit. b), an die werktägliche Arbeitszeit an. Diese Arbeitszeit darf auf bis zu zehn Stunden erhöht werden. Allerdings nur, wenn innerhalb eines Ausgleichszeitraums von sechs Monaten bzw. 24 Wochen acht Stunden pro Tag durchschnittlich nicht überschritten werden. Als Konsequenz aus der Anknüpfung des Arbeitszeitgesetzes an den Arbeitstag ergibt sich im Unterschied zur EU-Richtlinie Folgendes: Wird einmalig der Acht-Stunden-Tag um maximal zwei Stunden überschritten, so hat ein Ausgleich im vorgeschriebenen Zeitraum zu erfolgen. Dies wäre bei einer Anknüpfung an die Woche erst bei Überschreitung der Höchstarbeitszeitgrenze von 48 Stunden der Fall (vgl. Lohbeck 2001), die bei einer faktischen Fünf-Tage-Arbeitswoche viel mehr Überstunden voraussetzt.

Während der deutsche Anknüpfungspunkt an den einzelnen Arbeitstag für die Beschäftigten günstiger ist, steht der deutsche Ausgleichszeitraum von sechs Monaten im starken Widerspruch zur Arbeitszeitrichtlinie und dem dortigen Artikel 16 lit. b. Die Richtlinie sieht nämlich nur einen Ausgleichszeitraum von bis zu vier Monaten vor. Abweichungen von diesem Grundsatz sind staatlicherseits nur für besondere Tätigkeiten und mit zusätzlichen Gesundheitsvorkehrungen erlaubt (Artikel 17 Abs. 3 iVm 19 Abs. 1 der Richtlinie). Solche Regelungen sind aber im deutschen Arbeitszeitgesetz gerade nicht enthalten. Der lange Ausgleichszeitraum von sechs Monaten wird daher für europarechtswidrig gehalten (Buschmann/Ulber 2009, $\$ 3$ Rdnr. 12; Müller-Glöge et al. 2011, \$ 3 ArbZG Rdnr. 7 (Wank)). Konsequenz wäre nun eigentlich, dass der deutsche Gesetzestext im Sinne der Richtlinie ausgelegt wird. Der EuGH verbietet allerdings eine solche Auslegung dann, wenn ein Gesetzestext so eindeutig ist, dass gar kein Raum für eine Auslegung besteht (keine Auslegung contra legem, vgl. z. B. EuGH v. 4.7.2006, C-212/04 - Adeneler; Zwanziger 2007). Es bleibt abzuwarten, dass ein deutsches Gericht dieses Problem dem EuGH vorlegt, damit dieser eine Klärung herbeiführt. Für Arbeitsverhältnisse im öffentlichen Dienst gilt dagegen die (europäische) Viermonatsgrenze, da sich der Staat stets an EURichtlinien zu halten hat und sich nicht auf eine unzureichende Umsetzung berufen darf (Däubler et al. 2010, $\$ 3$ Rdnr. 18). Überhaupt wird das Arbeitszeitrecht im öffentlichen Dienst zukünftig allein deshalb unter Druck kommen, weil der EuGH jüngst (25.11.2010, C-429/09 - Fuß) einen Schadensersatzanspruch direkt aus der Richtlinie für den Fall abgeleitet hat, dass der Arbeitgeber hinsichtlich der Höchstarbeitszeit gegen Unionsrecht verstoßen hat (der Fall beurteilt Arbeitszeiten von beamteten Feuerwehrleuten).

Bei Verhandlungen, die Arbeitszeitregelungen schaffen, sollte insgesamt von dem Grundansatz aus $₫ 3$ ArbZG ausgegangen werden. Er gibt die Höchstgrenze der gesetzlich zulässigen täglichen Arbeitszeit vor. Und selbstverständlich können - dies soll ausdrücklich erwähnt werden
- tarifvertragliche oder einzelvertragliche Vereinbarungen auch kürzere Arbeitszeiten vorsehen. In der europäischen Arbeitswelt ist eine solche kürzere durchschnittliche Arbeitszeit, die in den vergangenen Jahren sogar rückläufig war, Realität: Die durchschnittliche Wochenarbeitszeit ging in der EU von 39 Stunden im Jahr 1990 auf 37,8 Stunden im Jahr 2006 zurück. Allerdings arbeiteten andererseits $10 \%$ aller Beschäftigten immer noch mehr als 48 Stunden pro Woche und nahezu $7 \%$ aller Beschäftigten stehen in mehr als einem Beschäftigungsverhältnis (vgl. EUKommission 2010).

\subsection{RUHEPAUSEN UND RUHEZEITEN}

Neben der werktäglichen Arbeitszeit gehören die Ruhepausen und die Ruhezeit ( $\$ \$ 4$ und 5 ArbZG) zu den allgemeinen Vorgaben des Arbeitszeitrechtes. Pausen werden von der Rechtsprechung als Freistellung der Beschäftigten von der Arbeit und von der Verpflichtung zum Bereithalten zur Arbeit verstanden (BAG v. 29.10.02, 1 AZR 603/01; BAG v. 22.07.03, 1 ABR 28/02). Sie sind auch während des Bereitschaftsdienstes, der Arbeitsbereitschaft und der Rufbereitschaft einzuhalten (BAG v. 5.6.03, 6 AZR 114/02). Die Länge der Pause beträgt 30 Minuten bei mehr als sechs Stunden Arbeitszeit und $45 \mathrm{Mi}$ nuten bei mehr als neun Stunden Arbeitszeit. Dabei kann sie in kleinere Einheiten von 15 Minuten aufgeteilt werden. Nach mehr als sechs Arbeitsstunden besteht eine Verpflichtung zur Ruhepause. Zweck der Ruhepausen ist in erster Linie die Erholung der Beschäftigten. Für besondere Beschäftigtengruppen gelten Sonderregelungen: Jugendliche haben 30 Minuten Pause nach 4,5 Stunden und ab 6 Stunden $60 \mathrm{Minu}$ ten Pause ( $\$ 11$ Jugendarbeitsschutzgesetz - JArbSchG); werdenden oder stillenden Müttern ist bei stehender Tätigkeit eine Sitzgelegenheit zum kurzen Ausruhen zu gewährleisten ( $\$ 2$ Abs. 2 Mutterschutzgesetz - MuSchG); für Fahrer, die unter die Richtlinie 51/2006/EG fallen, sind Lenkzeitunterbrechungen einzuhalten. Das Arbeitszeitgesetz regelt ausdrücklich keine Vergütungspflicht der Ruhepausen (Zwanziger 2007); diese entsteht nur ausnahmsweise durch einschlägige Tarif- oder Arbeitsvertragsregelungen.

Neben den Pausen enthält das Arbeitszeitgesetz in $\$ 5$ Vorgaben zur Ruhezeit. Eine Definition der Ruhezeit enthält es 
allerdings nicht. Die Arbeitszeitrichtlinie definiert sie als ,jede Zeitspanne außerhalb der Arbeitszeit" (Art. 2 Nr. 2). Dies wird qualitativ durch die Definition der ,ausreichenden Ruhezeiten“ in Artikel 2 Nr. 9 der Arbeitszeitrichtlinie ergänzt, welche erst dann vorliegen, wenn die Beschäftigten „über regelmäßige und ausreichend lange und kontinuierliche Ruhezeiten verfügen“" Nach dem deutschen Arbeitszeitgesetz erstreckt sich dieser Zeitraum über mindestens elf zusammenhängende Stunden ( $\$ 5$ Abs. 1 ArbZG). Darüber hinaus erlaubt das Gesetz eine Verkürzung der Ruhezeit auf zehn Stunden in Krankenhäusern, Pflegeeinrichtungen, Gaststätten, Verkehrsbetrieben, beim Rundfunk sowie in der Landwirtschaft, wenn jede Verkürzung der Ruhezeit innerhalb von vier Wochen durch Verlängerung einer anderen Ruhezeit auf mindestens zwölf Stunden ausgeglichen wird ( $\$ 5$ Abs. 2 ArbZG). Während der Inanspruchnahme in der Rufbereitschaft sieht $\$ 5$ Abs. 3 ArbZG eine weitere Verkürzung der Ruhezeit in Krankenhäusern und Pflegeeinrichtungen vor. Die Verkürzung der Ruhezeit darf nur um die Hälfte erfolgen. Das heißt, 5,5 Stunden Ruhezeit müssen erhalten bleiben.

\subsection{NACHT- UND SCHICHTARBEIT}

$\$ 6$ ArbZG regelt die Grundsätze der Nacht- und Schichtarbeit. Anknüpfend an die grundlegende Rechtsprechung des BVerfG zur Nachtarbeit aus dem Jahr 1992 muss bei der Ausübung von Nacht- und Schichtarbeit das Recht auf körperliche Unversehrtheit unbedingt gewahrt sein. Diesem Grundsatz trägt die deutsche Regelung nur unzureichend Rechnung. Schon in Absatz 1 des $\$ 6$ ArbZG wird dies durch eine wenig konkrete Festlegung deutlich, wenn es dort heißt: ,,nach gesicherten arbeitswissenschaftlichen Erkenntnissen über die menschengerechte Gestaltung der Arbeitszeit von Nacht- und Schichtarbeitern". Dies steht im Widerspruch zu Artikel 12 der Arbeitszeitrichtlinie, der den Gesetzgeber verpflichtet, zum Schutz von Schicht und Nachtarbeitern vollständige Grundsätze zu regeln (EuGH v. 1.5.2005, C-14/04 - Dellas). Verstößt ein Arbeitgeber gegen die menschengerechte Gestaltung der Arbeit, so ist dies im deutschen Recht nicht mit Sanktionen verknüpft ( $\$ 6$ Abs. 1 ArbZG ist nicht im Bußgeldkatalog des $\$ 22$ ArbZG aufgeführt), wodurch die Effektivität der Vorschrift abermals unterspült wird. Hinzu kommt, dass sehr umstritten ist, was "gesicherte arbeitswissenschaftliche Erkenntnisse“ sind. Anerkennung vor Gericht haben gefunden: die Leitlinien für Schichtarbeiter der Europäischen Stiftung zur Verbesserung der Lebens- und Arbeitsbedingungen, ${ }^{1}$ die Veröffentlichungen der Bundesanstalt für Arbeitsschutz und Arbeitsmedizin (BAuA) zum Thema Schicht- und Nachtarbeit ${ }^{2}$ sowie die Leitlinien der deutschen Gesellschaft für Arbeitsmedizin und Umweltmedizin e.V. (DGAUM) zu Nacht- und Schichtarbeit. ${ }^{3}$ Ein Nachtarbeitsverbot besteht grundsätzlich für Jugendliche, werdende und stillende Mütter ( $\$ \$ 14$ und 18 JuSchG und $\$ 8$ Abs. $1 \mathrm{MuSchG}$ ).

Oft vergessen wird allerdings eine Besonderheit für Nachtarbeiter: $\$ 6$ Abs. 5 ArbZG formuliert einen einklagbaren Anspruch auf eine angemessene Zahl bezahlter freier Tage oder einen angemessenen Zuschlag auf das Arbeitsentgelt. Dies sollte in entsprechenden Tarifverträgen bzw. Betriebsvereinbarungen immer flankierend aufgenommen werden (BAG v. 5.9.2002, 9 AZR 202/01; vgl. zur Angemessenheit des Zuschlags Henssler et al. 2010, § 6 ArbZG Rdnr. 20 (Gäntgen)).

Neben der Gestaltung der Arbeitszeit von Nacht- und Schichtarbeitern regelt das Gesetz deren tägliche Arbeitszeit in $\$ 6$ Abs. 2 ArbZG. Hier darf es in einem vierwöchigen Ausgleichszeitraum nicht zu längeren Arbeitszeiten als acht Stunden pro Werktag kommen. Zudem haben Nachtarbeiter das Recht auf regelmäßige arbeitsmedizinische Untersuchungen, aufgrund derer ihnen unter gegebenen Umständen ein Recht auf Umsetzung in den Tagdienst zustehen kann ( $\$ \$ 6$ Abs. 3 und 4 ArbZG). Neben der medizinischen Indikation besteht außerdem aufgrund von Betreuungspflichten oder Pflegebedürftigkeit von Angehörigen eine Umsetzungsmöglichkeit in den Tagdienst. Zuletzt hat der Arbeitgeber für die Nachtarbeiter dieselben Weiterbildungsangebote und aufstiegsfördernden Maßnahmen zu sichern, wie für alle anderen Beschäftigten auch ( $\$ 6$ Abs. 6 ArbZG). Der Arbeitgeber hat hierzu gegebenenfalls den Schichtplan umzustellen oder die Beschäftigten für die Weiterbildung in den Tagdienst umzusetzen (vgl. Buschmann/ Ulber 2009, \$ 6 Rdnr. 31).

Ist der Beschäftigte arbeitsvertraglich verpflichtet, eine bestimmte Anzahl von Stunden pro Tag (mit festgelegten Pausen- und Ruhezeiten) oder Nacht- und
Schichtarbeit zu leisten, kann der Arbeitgeber die Umstände hierzu mithilfe seines Direktionsrechts bestimmen. $\$ 106$ Gewerbeordnung (GewO) sieht allerdings Einschränkungen des Direktionsrechts durch Betriebsvereinbarung, Tarifverträge oder gesetzliche Vorschriften vor. Das Direktionsrecht findet somit auch seine Grenzen in der erzwingbaren Mitbestimmung. Verstößt der Arbeitgeber gegen arbeitsschutzrechtliche Bestimmungen, erwächst dem Beschäftigten ein Leistungsverweigerungsrecht. Die Grenze des Direktionsrechts ist in Anwendung des AllgemeinenGeschäftsbedingungen-Rechts auch dann gegeben, wenn der Arbeitsvertrag Klauseln zur Arbeitszeit enthält, die eine unangemessene Benachteiligung im Sinne von $\$ 307$ Abs. 2 Nr. 1 Bürgerliches Gesetzbuch (BGB) darstellen. Hiermit ist zum Beispiel die Vereinbarung überlanger täglicher oder sogar nächtlicher Arbeitszeiten gemeint (vgl. auch Däubler et al. 2008, § 307 BGB Rdnr. 179f.).

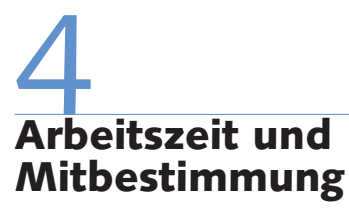

Diese gesetzlichen Vorgaben zur Arbeitszeit werden flankiert durch Mitbestimmungsrechte. Zur Vereinfachung wird hier zunächst auf die Mitbestimmungsrechte des Betriebsrates eingegangen. Die entsprechenden Rechte der Personalräte und Mitarbeitervertretungen im kirchlichen Bereich sind in Tabelle 1 aufgelistet.

$\$ 87$ Abs. 1 Nr. 2 Betriebsverfassungsgesetz (BetrVG) legt die Mitbestimmungsrechte der Betriebsräte bei Beginn und Ende der täglichen Arbeitszeit einschließlich der Lage der Pausen fest. Darunter fällt auch die Verteilung der Arbeitszeit auf die einzelnen Wochentage. $\$ 87$ Abs. 1 Nr. 3 BetrVG regelt die Mitbestimmung bei vorübergehender Verkürzung oder Verlängerung der betriebsüblichen $\mathrm{Ar}$ beitszeit. Damit sind sowohl die Verlängerung der täglichen Arbeitszeit auf zehn Stunden als auch der Ausgleichszeitraum bei Verlängerung des Acht-Stunden-Tages

\begin{tabular}{ll}
\hline 1 & http://eurofound.europa.eu/. \\
\hline 2 & http://www.baua.de/cln_135/sid_1FB30A1AC2 \\
& 480E328EB601488D97531F/de/Publikationen/ \\
& AWE/AWE.html. \\
\hline 3 & http://www.dgaum.de/.
\end{tabular}




\section{Tabelle 1: Mitbestimmungs- und Beteiligungsrechte bei der Arbeitszeit}

\begin{tabular}{|c|c|c|c|}
\hline & Betriebsrat (BetrVG) & Personalrat (BPersVG) & Mitarbeitervertretung (MVG-EKD) \\
\hline Beginn und Ende der täglichen Arbeitszeit & $\S 87$ Abs. 1 Nr. 2 & $\S 75$ Abs. 3 Nr.1 & $\S 40$ lit. $d$ \\
\hline Pausen & $\S 87$ Abs. 1 Nr. 2 & $\S 75$ Abs. 3 Nr.1 & $\S 40$ lit. d \\
\hline Verkürzung/Verlängerung der Arbeitszeit & $\S 87$ Abs. 1 Nr. 3 & $\S 75$ Abs. 4 & Keine Regelung \\
\hline Nacht- und Wechselschicht & $\S 87$ Abs. 1 Nr. 2,7 und 10 & $\S 75$ Abs. 3 Nr. 4 und 11 & $\S 40$ lit. d \\
\hline
\end{tabular}

zustimmungspflichtig und ohne die $\mathrm{Zu}$ stimmung des Betriebsrates unzulässig (Theorie der Wirksamkeitsvoraussetzung; vgl. Henssler et al. 2010, $\$ 87$ BetrVG, Rdnr. 38 (Clemenz); Däubler et al. 2008, $\S 87$ Rdnr. 4). Das Mitbestimmungsrecht scheidet allerdings aus, sobald ein Tarifvertrag besteht $(\$ 87$ Abs. 1 Einleitungssatz BetrVG). Wenn der Betriebsrat zum Beispiel zur Länge des Ausgleichszeitraumes eine Betriebsvereinbarung abschließen möchte, muss dabei die tarifvertragliche Regelung berücksichtigt werden. Möchte der Betriebsrat über die gesetzliche Pausenregelung hinaus weitere Pausenregelungen für den Betrieb vorsehen, so kann er dies durch Abschluss einer Betriebsvereinbarung tun. Allerdings ist dies nach der Rechtsprechung (BAG v. 1.7.2003, 1 ABR 20/02) nur hinsichtlich der zeitlichen Lage der Pausen, nicht aber bezogen auf die Vereinbarung einer Vergütungspflicht für die Pausen möglich.

Bei Nacht- und Schichtarbeit ist zu unterscheiden zwischen der Einführung und Ausgestaltung sowie der Überwachung der Durchführung dieser Arbeitsform. Mitbestimmungsrechte aus $\$ 87$ Abs. 1 Nr. 2 BetrVG bestehen dann, wenn Nacht- und Schichtarbeit eingeführt werden (Fitting et al. 2010, $\$ 87$ BetrVG Rdnr. 120). Diese umfassen nach der Rechtsprechung (BAG v. 28.10.1986, 1 ABR 11/85) den fest $\mathrm{zu}$ legenden Personenkreis, die zeitliche Lage der Schichten und die Form des Ausgleichs für die tatsächliche Ableistung von Nachtschichten. Gerade bei der Nacht- und Schichtarbeit hat der Betriebsrat gemeinsam mit dem Arbeitgeber seine Pflicht zur „menschengerechten Gestaltung“ dieser Tätigkeit zu erfüllen. Es sind deshalb in Betriebsvereinbarungen detaillierte Regelungen zur Ausgestaltung der Nacht- und Schichtarbeit im Betrieb zu schaffen. Auch die Änderung der Nacht- und Schichtarbeit unterliegt der Mitbestimmung (ebd., Rdnr. 121).Zusätzlich kann der Betriebsrat in Ausübung seiner Überwachungspflichten die Einhaltung der arbeitswissenschaftlichen Erkenntnisse verlangen $(\$ 80$
Abs. 1 Nr. 1 BetrVG). In Anwendung von $\$ 87$ Abs. 1 Nr. 7 BetrVG kann er auch eigene Vorschläge zum Arbeits- und Gesundheitsschutz der Nacht- und Schichtarbeiter machen.

Über die Entscheidung, ob der Ausgleich für Nachtarbeit in Form von Entgelt oder Freizeit erfolgen soll, besteht ein Mitbestimmungsrecht nach $\$ 87$ Abs. 1 Nr. 7 und 10 BetrVG. Dieses endet allerdings bei der Bestimmung der Höhe des Zuschlags oder des Zeitumfangs des Ausgleichs (BAG v. 26.8.1997, 1 ABR 16/97).

\section{Abweichende Regelungen}

$\$ 7$ ArbZG erlaubt den Tarifvertragsparteien umfangreiche Abweichungsmöglichkeiten von den dargestellten Grundregeln. Man könnte auch formulieren, dass damit den Tarifparteien bzw. aufgrund eines Tarifvertrages auch den Betriebsparteien das Arbeitszeitrecht weitestgehend überlassen wird. Dies hat positive wie negative Seiten. Der Staat entfernt sich damit von seiner verfassungsrechtlich bestehenden Hüterfunktion hinsichtlich des Arbeits- und Gesundheitsschutzes. Zudem werden dadurch Tendenzen einer Ökonomisierung im Arbeitszeitrecht gefördert - trotz aller Bemühungen der Interessenvertretungen -, weil das Arbeitszeitrecht Teil der „Verhandlungsmasse" bei Verhandlungen um Tarifverträge bzw. Betriebsvereinbarungen wird. Angesichts der Zielsetzung des Arbeitszeitrechts darf sich der Staat nicht völlig zurückziehen - Verhandlungen zu Tarifverträgen und Betriebsvereinbarungen müssen sich vom Prinzip des Gesundheitsschutzes leiten lassen. Seit 2004 eröffnet im Übrigen das Arbeitszeitgesetz Abweichungsmöglichkeiten auch durch Dienstvereinbarungen. Damit ist nach der Privatwirtschaft auch der öffentliche Dienst den gleichen Abweichungsregelungen unterworfen.

\section{1 § 7 ABS. 1 UND ABS. 2 ARBZG}

$\$ 7$ Abs. 1 Nr. 1 a ArbZG sieht für den Fall, dass Arbeitsbereitschaft oder Bereitschaftsdienst in erheblichem Umfang geleistet wird, eine Arbeitszeitverlängerung über zehn Stunden werktäglich vor. Der Ausgleichszeitraum (um 48 Stunden im Durchschnitt zu erreichen) wird von sechs auf zwölf Monate verlängert ( $\$ 7$ Abs. 8 ArbZG). Die EU-Richtlinie dagegen erlaubt - wie erwähnt - einen grundsätzlichen Ausgleichszeitraum von nur vier Monaten (Art. 16) und gestattet Abweichungsmöglichkeiten bis zu max. sechs Monaten nach Art. 19 Abs. 1 bzw. bis zu zwölf Monaten nur in ganz eng begrenzten Ausnahmefällen (Art. 19 Abs. 2 der Richtlinie). Die Tarifparteien (Betriebsparteien) sollten deshalb in Verhandlungen von einem überlangen Ausgleichszeitraum in der Regel absehen bzw. sich streng an die Vorgaben aus der Richtlinie halten. Neben diesem Fall regelt $\$ 7$ Abs. 1 ArbZG Abweichungsmöglichkeiten von den Ruhepausen in Schicht- und Verkehrsbetrieben, von den Ruhezeiten nach $\$ 5$ Abs. 1 ArbZG sowie der Regelung der Nachtarbeit im Sinne von $\$ 6$ Abs. 2 ArbZG.

Schließlich erlaubt $\$ 7$ Abs. 2 ArbZG für bestimmte Branchen oder Dienste weitere Abweichungen von den Arbeitszeitregelungen. So kann bei Ruhezeit, Nachtarbeit und täglicher Höchstarbeitszeit von den Grundregeln abgewichen werden, wobei Kürzungen der Ruhezeit nach $\$ 7$ Absatz 2 Nr. 1 ArbZG nicht mehr bei Bereitschaftsdienstzeiten, sondern nur noch bei Rufbereitschaft möglich sind. Zusätzlich ist dem Gesundheitsschutz der Beschäftigten dadurch Rechnung zu tragen, dass ein entsprechender Zeitausgleich gewährleistet wird. Ein anderer Ausgleich, etwa in Geld, ist ausdrücklich nicht vorgesehen (Henssler et al. 2010, $\$ 7$ ArbZG Rdnr. 10 (Gäntgen)).

5.2 SPEZIALFALL § 7 ABS. 2A ARBZG

Anders als die beiden ersten Absätze von $\$ 7$ ArbZG ermöglicht Absatz 2a eine Ar- 
beitszeitverlängerung ohne Ausgleich (dies gilt für die Höchstarbeitszeiten, Nachtarbeitszeiten und Ruhezeitverkürzungen), wenn in die Arbeitszeit regelmäßig und in erheblichem Umfang Arbeitsbereitschaft oder Bereitschaftsdienst fällt (als Richtwert $30 \%$; vgl. Anzinger/Koberski 2009, § 7 Rdnr. 23). Die Verlängerungsmöglichkeit ist nach oben unbegrenzt möglich, weil $\$ 7$ Abs. 8 ArbZG (Begrenzung auf 48 Stunden) nicht anzuwenden ist. Voraussetzung ist eine vorherige schriftliche Einwilligung der Beschäftigten, die mit einer Frist von sechs Monaten widerrufen werden kann ( $\$ 7$ Abs. 7 ArbZG). Die Tarifvertragsoder Betriebsparteien haben zudem laut Gesetzestext durch besondere Regelungen sicherzustellen, dass die Gesundheit der Beschäftigten durch die Verlängerung der Arbeitszeit nicht gefährdet wird. Art. 18 der EU-Richtlinie, der die Abweichungen im Wege von Tarifverträgen abschließend behandelt, enthält aber gar keinen Verweis auf Art. 6 der Richtlinie, der die wöchentliche Höchstarbeitszeit regelt. Das europäische Arbeitszeitrecht wird hier erneut gebrochen (ebenfalls kritisch: Müller-Glöge et al. 2011, $\$ 7$ ArbZG 18 (Wank)).

$\$ 7$ Abs. 2a ArbZG kann also für den einzelnen Beschäftigten zu Belastungen führen, die das Gesetz nach seiner sonst bestehenden Gesamtkonzeption gerade verhindern will. Auch die Einwilligung zur Arbeitszeitverlängerung ist kein Schutz vor (Selbst-)Ausbeutung, da sie oft unter Druck gegeben wird. Zu Art. 17 der Vorgängerrichtlinie 94/103/EG ist durch den EuGH bereits klargestellt worden, dass die Nutzung von Abweichungsmöglichkeiten auf das „unbedingt Erforderliche“ zu beschränken ist (EuGH v. 9.9.2003, C-151/02 - Jaeger). Noch enger müssen doch dann die Spielräume sein, wenn es um das völlige Aushebeln der Grenzen zur Höchstarbeitszeit geht - und auch dies hat der EuGH im zitierten Urteil festgelegt. Die deutsche Gesetzeskonzeption geht daher zu weit. Art. 22 der EU-Richtlinie, der das berühmt-berüchtigte „individuelle Opt-out" und die Möglichkeit zur Ausweitung der Höchstarbeitszeiten enthält, hat zudem allein den Staat als Adressaten. Er muss garantieren, dass die Grundsätze der Sicherheit und des Gesundheitsschutzes der Beschäftigten eingehalten werden. Es war daher dem deutschen Gesetzgeber im EU-Sinn verwehrt, so weitreichende Kompetenzen auf die Tarifvertragsparteien zu übertragen (vgl. Buschmann/
Ulber 2009, $\$ 7$ Rdnr. 24c; Däubler et al. 2010, $\$ 7$ Rdnr. 21). Überdies ist es europarechtlich zu beanstanden, dass $\$ 7$ Abs. 2a ArbZG neben der Arbeitszeitverlängerung auch die Ruhezeiten nach $\$ 5$ Abs. 1 ArbZG nennt (vgl. auch Lörcher/Jerchel 2004, S. 38f.), diese sind von der europäischen Opt-out-Regelung ausdrücklich nicht umfasst. Kürzungen der Ruhezeiten sind damit europarechtlich eindeutig ausgeschlossen. Schließlich verbietet Art. 23 der EU-Richtlinie eine Zurücknahme des allgemeinen Arbeitnehmerschutzes im Zuge der Umsetzung der Richtlinie in nationales Recht.

\subsection{REGELUNGEN FÜR DIE KIRCHEN}

Eine „Steigerung“ enthält $\$ 7$ Abs. 4 ArbZG für den Bereich der Kirchen und öffentlich-rechtlichen Religionsgemeinschaften. Diese nutzen in Deutschland umfangreich ihren Spielraum aus den Artikeln 140 GG, 137 WRV (Selbstbestimmungsrecht). Im sogenannten Dritten Weg werden dabei Arbeitsvertragsrichtlinien entwickelt, an deren Entstehung Gewerkschaften nicht mitwirken. Diese dürfen nun ebenfalls abweichende Regelungen zu den Kernvorschriften des ArbZG enthalten. $\$ 7$ Abs. 4 ArbZG ist bedenklich im Kontext von Art. 18 der EU-Arbeitszeitrichtlinie, der Abweichungen im Wege von Tarifverträgen oder Betriebsvereinbarungen anspricht. Die einseitigen Kirchenregelungen sind hiervon aber sicher nicht umfasst. Entgegen der Rechtsprechung des EuGH können die Kirchen sogar festlegen, dass Bereitschaftsdienste nicht der Arbeitszeit zugerechnet werden (Henssler et al. 2010, $\$ 7$ ArbZG Rdnr. 16 (Gäntgen)). Vor dem Hintergrund zahlreicher kirchlich geführter Krankenhäuser (Diakonie/Caritas) ist dies sehr bedenklich.

\subsection{AUSGLEICH FÜR SONN- UND FEIERTAGSBESCHÄFTIGUNG}

Für den Ausgleich von Sonn- und Feiertagsbeschäftigung sieht das Gesetz in $\$ 11$ ArbZG besondere Regelungen vor, von denen nach $\$ 12$ ArbZG durch oder aufgrund Tarifvertrags abgewichen werden kann. Hier sei nur auf eine besondere gesundheitsgefährdende Variante hingewiesen: Nach $\S 11$ Abs. 3 ArbZG ist für Sonntagsarbeit ein Ersatzruhetag innerhalb von zwei Wochen vorgesehen. Die Rechtsprechung lässt es zu, dass dieser Ersatzruhetag bereits dann abgegolten bzw. gewährt ist, wenn innerhalb der zwei Wochen ein Tag ohnehin frei ist. In diesen Fällen wird also kein weiterer Tag gewährt (BAG v. 23.3.2006, 6 AZR 497/05; zu Recht anderer Ansicht: Anzinger/Koberski 2009, \$11 Rdnr. 23; Buschmann/Ulber 2009, $\$ 11$ Rdnr. 6a). Nutzt man dies als Arbeitgeber "geschickt“, kann man Beschäftigte bis zu einer Dauer von 19 Tagen (!) am Stück einsetzen.

Ein Beispiel aus der Straßenreinigungsbranche: Eine Person arbeitet grundsätzlich an sechs Tagen in der Woche. In der ersten Woche wird sie zusätzlich mit Sonntagseinsatz belegt. Erst nach weiteren 13 Arbeitstagen liegt dann der Ersatzruhetag, ein Sonntag, der ohnehin frei ist, was aber nach der angegebenen Rechtsprechung ausreicht.

Wir halten dies für bedenklich, zumal das BAG in der Entscheidung vom 23.3.2006 eben auch formuliert (Rz. 16): „Im Vordergrund steht der Arbeitsschutz $(\$ 1$ ArbZG). $\$ 11$ Abs. 3 ArbZG dient in erster Linie dem Gesundheitsschutz der Arbeitnehmer aus öffentlichem Interesse. Der Arbeitnehmer soll mindestens einen Ruhetag pro Sieben-Tage-Zeitraum haben".

\section{0 \\ Folgerungen aus der Gesetzeslage}

Obwohl das deutsche Arbeitszeitrecht weite Ausnahmen erlaubt, sollten Gewerkschaften und Betriebsräte insbesondere von $\$ 7$ Abs. 2a ArbZG in der Regel keinen Gebrauch machen, und zwar nicht nur wegen rechtlicher Überlegungen, sondern vor allem, weil die dortigen Möglichkeiten klar zulasten der Gesundheit der Beschäftigten gehen. Allenfalls sind Abweichungen akzeptabel, wenn zuvor ausgleichende Gesundheitsschutzmaßnahmen mit vereinbart, durchgeführt und dokumentiert werden. Das gilt insbesondere für z. B. die Gesundheitsbranche, den Feuerwehrbereich, die Verkehrsberufe, die Medien sowie den Gastronomiesektor, die teilweise durchaus nachvollziehbare spezifische Arbeitszeitbedürfnisse haben. Es ist daher zu empfehlen, in einem ersten Schritt vom Arbeitgeber eine Gefährdungsanalyse für jeden einzelnen Arbeitsplatz nach $\$ 5 \mathrm{Ar}$ beitsschutzgesetz (ArbSchG) einzufordern, und zwar bevor über eine Arbeitszeitverlängerung verhandelt wird. 


\subsection{FOLGERUNGEN FÜR TARIFPARTEIEN}

$\$ 7$ Abs. 2a ArbZG mag europarechtlich bedenklich sein, in der Praxis wird gleichwohl von ihm Gebrauch gemacht. Hierzu hat jüngst das Bundesarbeitsgericht (BAG) (23.6.2010, AZR 543/09) Vorgaben gemacht, die die Tarifvertragsparteien zu beachten haben. Für den Bereich Werksfeuerwehr hat das BAG entschieden, dass die für diese Branche typischen und wiederkehrenden 24-Stunden-Dienste, hier geregelt im Manteltarifvertrag für die Chemische Industrie West, zulässig und von $\$ 7$ Abs. 2a ArbZG gedeckt sind - (nochmals: obwohl Art. 22 der EU-Richtlinie eine Ausweitung der Höchstarbeitszeiten durch Tarifverträge gar nicht gestattet). Trotz der Erwähnung der einschlägigen Rechtsprechung des EuGH im Urteil hat sich das BAG aber nicht veranlasst gesehen, die Sache dem EuGH nach Art. 267 AEUV vorzulegen. Immerhin - und das betrifft nun die Tarifvertragsparteien hat es für die flankierenden Regelungen zur Sicherstellung der Gesundheit der Arbeitnehmer in Fällen der Ausweitung der Höchstarbeitszeit ohne Ausgleich festgelegt: „Der Tarifvertrag muss beispielsweise zusätzliche Pausenvorschriften, besondere Ruhezeitregelungen oder spezielle arbeitsmedizinische Maßnahmen enthalten“. Tarifvertragliche Regelungen müssen also hinreichend konkret sein; pauschale und allgemeine Bekenntnisse für einen guten Gesundheitsschutz genügen nicht.

\subsection{FOLGERUNGEN FÜR BETRIEBSPARTEIEN}

Die betrieblichen Verhandlungen über eine Arbeitszeitregelung bedürfen, um zu einem effektiven Arbeits- und Gesundheitsschutz zu gelangen, einer intensiven Vorbereitung. Die Interessenvertretungen sollten sich also Zeit nehmen und die Beschäftigten rechtzeitig einbeziehen. Vor der Aufnahme der Verhandlungen muss eine Bestandsaufnahme bestehender betrieblicher Arbeitszeiten und -formen erstellt werden. Zudem sind mögliche unterschiedliche Regelungsbedarfe in Betriebsteilen zu prüfen. Es ist überdies nach unserer Meinung unerlässlich, vor Beginn jeder Verhandlung, aber auf jeden Fall vor Verhandlungsabschluss, eine Gefährdungsanalyse nach $\$ 5$ ArbSchG über die gesundheitlichen Belastungen an je- dem einzelnen Arbeitsplatz für die jeweilige Beschäftigtengruppe durchzuführen. ${ }^{4}$ In fast allen Phasen von Vorbereitung und Verhandlung selbst hat sich das Hinzuziehen von betriebsexternem Sachverstand durch den Betriebsrat bewährt.

Konkrete spezifische Inhalte einer solchen Vereinbarung hängen von den jeweiligen betrieblichen Besonderheiten in Bezug auf Arbeitsabläufe und die jeweilige betriebliche Organisation ab. Sie sind aber in jedem Fall den rechtlichen Vorgaben unterworfen, die oben umfassend dargestellt wurden. Notwendige Regelungen in einer Arbeitszeitvereinbarung sollte es geben zu:

- Grundsätzen zur Arbeitszeitgestaltung und der Planung von Dienst- oder Schichtplänen inklusive erforderlicher Maßnahmen zum Arbeits- und Gesundheitsschutz; - Beginn und Ende der täglichen Arbeitszeit sowie der Lage der Pausen;

- Benennung von Verantwortlichen für die Einhaltung eines Schicht- oder Dienstplans;

- Höchstgrenzen der Arbeitszeit pro Tag oder Woche;

- Konkrete Bezifferung der Ausgleichszeiträume bei Überschreitung der gesetzlich zulässigen Höchstarbeitszeit;

- Gestaltung der Nachtarbeit;

- Elektronische Zeiterfassung, soweit eine solche noch nicht existiert;

- Behandlung von Wasch- und Umkleidezeiten;

- Entgeltfragen.

Auf die Einhaltung der allgemeinen Grundsätze der Sicherheit und des Gesundheitsschutzes im oben beschriebenen Sinne hat auch die Einigungsstelle zu achten (Däubler et al. 2010, \$ 7 Rdnr. 21).

Bestehen bereits betriebliche Vereinbarungen zur Arbeitszeit und hält sich der Arbeitgeber nicht ordnungsgemäß daran oder verstößt er gegen mitbestimmungspflichtige Arbeitszeitvorgaben, kann der Betriebsrat die Unterlassung vereinbarungswidriger Maßnahmen verlangen. Auf seinen Antrag kann das Arbeitsgericht im Falle einer Zuwiderhandlung ein Ordnungsgeld in Höhe von bis zu $10.000 €$ androhen (BAG v. 5.10.2010, 1 ABR 71/09). In diesem jüngst entschiedenen Fall hatte die Arbeitgeberin gegen eine bei ihr geltende Betriebsvereinbarung zur Arbeitszeit verstoßen. Auf Antrag des Betriebsrats hatten die Vorinstanzen ihr aufgegeben, es zu unterlassen, Mitarbeiter ohne Zustimmung des Betriebsrats aus der Zeiterfassung herauszunehmen. Das Bundesarbeitsgericht bestätigte dies insoweit (das Gericht nahm an anderer Stelle Einschränkungen vor), als der Arbeitgeberin für den Fall des Zuwiderhandelns ein Ordnungsgeld in Höhe von $10.000 €$ angedroht wurde. Mithilfe dieses Anspruchs aus $\$ 23$ Abs. 3 BetrVG hat der Betriebsrat ein wirksames Instrument auch zur Durchsetzung von mitbestimmungspflichtigen Regelungen der Arbeitszeit.

\section{Ausblick und Gefahren}

Nachdem im Jahr 2009 unter anderen politischen Mehrheiten eine Änderung der EUArbeitszeitrichtlinie gescheitert war, hat die EU-Kommission im März des Jahres einen erneuten Vorstoß unternommen und sich mit dem Ziel an die Sozialpartner gewandt, im Wege des Konsultationsverfahrens Verhandlungen über eine Änderung der Arbeitszeitrichtlinie aufzunehmen (EU-Kommission 2010). Die Kommission regt an, über folgende Punkte zu diskutieren:

- Dauerhafte Beibehaltung des sogenannten individuellen Opt-out;

- Anhebung des Ausgleichszeitraums zur durchschnittlichen Berechnung der 48-Stunden-Grenzen von vier auf zwölf Monate;

- Höhere Flexibilität bei der Lage der täglichen und wöchentlichen Ruhezeiten;

- Einordnung der Bereitschaftsdienste in solche, die als Arbeitszeit gelten und solche, die nicht hierunter fallen (sog. aktive und inaktive Zeiten).

Sehr kritisch dürfte zu sehen sein, dass die Feststellungen und Anregungen der Kommission die Tendenz zeigen, Gesundheitsbelange zu niedrig zu bewerten. $\mathrm{Zu}$ wenig werden die Vorgaben der Art. 31 der EUGrundrechte-Charta und Art. 151 AEUV in die Überlegungen einbezogen. Der Europäische Gewerkschaftsbund hat u.a.

4 Vgl. z.B. die Muster und eine entsprechende Checkliste für den Pflegedienst Völpel-Haus/ Lechler (2008) sowie diesbezüglich BAG v. 23.6.2010, 10 AZR 543/09. In dieser Entscheidung sieht das BAG es sogar für unzureichend an, wenn im Bereich des § 7 Abs. 2a ArbZG Maßnahmen lediglich nach $\S 5$ ArbSchG ergriffen werden. 
deshalb schon im Rahmen des inzwischen begonnenen Konsultationsverfahrens erklärt, dass es eine Einigung zwischen den Sozialpartnern auf Basis der Vorschläge der Kommission aus seiner Sicht nicht geben werde, weil die Interessen der Sozialpartner zu weit auseinander liegen (vgl. Schubert/ Jerchel 2009/2010). Im Dezember 2010 leitete die EU-Kommission die zweite Konsultationsphase zur Überarbeitung der EU-Arbeitszeitpolitik ein. Die Sozialpartner haben noch bis Ende Februar 2011 die Gelegenheit, ihre Ansichten zu den verschiedenen Änderungsoptionen darzulegen (vgl. Europäische Union (Hrsg.): IP/10/1760 vom 21.12.2010 mit weiteren Verweisen). ${ }^{5}$

Unübersehbar ist die Gefahr, dass sich im Arbeitszeitrecht immer mehr Interessen der Arbeitgeber durchsetzen und die der Arbeitnehmer, insbesondere der Gesundheitsschutz, dahinter weiter zu- rück bleiben. Mit ihrem Vorstoß will die Kommission unseres Erachtens die richtigen früheren Entscheidungen des EuGH, insbesondere $\mathrm{zu}$ Bereitschaftsdiensten, ins Leere laufen lassen. Die Bundesregierung ist aufgefordert, falls die Kommission bei ihrer Initiative zur Änderung der Arbeitszeitrichtlinie bleibt, im Rat für einen ausreichenden Gesundheitsschutz im Arbeitszeitrecht zu plädieren. Sollte die Bundesregierung dies unterlassen oder die Kommission sich letztlich durchsetzen, müssten Gewerkschaften und Betriebsräte durch verbleibende Handlungsmöglichkeiten gerade dem Gesundheitsschutz Raum verschaffen.

Eines muss zum Abschluss eingeräumt werden: Spricht man, wie auch dieser Beitrag, von Handlungsspielräumen für Interessenvertretungen, so wird deutlich, dass es eigentlich um einen Abwehrkampf gegen arbeitgeberseitig veranlasste und vom
Gesetzgeber unterstützte nachteilige neue Arbeitszeitregelungen geht. Dabei wird der Aspekt des Gesundheitsschutzes schon von der heutigen deutschen Gesetzeslage stark unterbewertet. Kritiker werden vielleicht einwenden, es gebe doch auch einen positiven Handlungsspielraum für Interessenvertretungen, wenn man beispielsweise an Langzeitkonten-Systeme denkt. Ja und nein, denn Langzeitkonten-Systeme können sich unserer Ansicht nach nur innerhalb des zulässigen Arbeitszeitrahmens wie beschrieben abspielen. Systeme, die Anreize zur Selbstausbeutung geben, also z. B. $\$ 7$ Abs. 2a ArbZG voll ausnutzen, sind vorsichtig zu betrachten. Bei diesen geht der Beschäftigte mit seiner Gesundheit in Vorleistung

5 http://europa.eu/rapid/pressReleasesAction.do? reference $=I P / 10 / 1760$ \& format $=H T M L \&$ aged $=0$ \&language $=\mathrm{DE}$.

\section{LITERATUR}

Anzinger, R./Koberski, W. (2009): Kommentar zum Arbeitszeitgesetz, Frankfurt/M.

Buschmann, R./Ulber, J. (2009): Basiskommentar Arbeitszeitgesetz mit Nebengesetzen und Ladenschluss, Frankfurt/M.

Däubler, W./Dorndorf, E./Bonin, B./Deinert, O. (2008): AGB-Kontrolle im Arbeitsrecht, München

Däubler, W./Kittner, M./Klebe, T. (2008): Kommentar zum BetrVG, Frankfurt/M.

Däubler, W./Hjort, J.P./Schubert, M./Wolmerath, M. (2010): Handkommentar Arbeitsrecht, Baden-Baden

EU-Kommission (Hrsg.) (2010): Zur Überarbeitung der Arbeitszeitrichtlinie vom 24.03., $\operatorname{KOM}(2010), 106$ endg., Brüssel

Fitting, K./Engels, G./Schmidt, I./Trebinger, Y./Linsenmaier, W.

(2010): Betriebsverfassungsgesetz: BetrVG, München

Henssler, M./Willemsen, H. J./Kalb, H. J. (2010): Arbeitsrecht Kom-

mentar, Köln
Lörcher, K./Jerchel, K. (2004): Bereitschaftsdienst ist Arbeitszeit, hrsg. vom ver.di Bundesvorstand, Berlin

Lohbeck, A. (2001): Arbeitszeitrechtliche Rahmenbedingungen bei der Gestaltung betrieblicher Arbeitszeitmodelle, in: Zeitschrift für Tarifrecht (ZTR) 8, S. 342-349

Müller-Glöge, R./Preis, U./Schmidt, I. (2011): Erfurter Kommentar zum Arbeitsrecht, München

Schubert, J./Jerchel, K. (2010): Das europäische Arbeitsrecht, in: Europäische Zeitschrift für Wirtschaftsrecht (EuZW) (18), S. $687-693$

Völpel-Haus, D./Lechler, N. (2008): Dienstplangestaltung im Pflegedienst, hrsg. vom ver.di Bundesvorstand, Berlin

Zwanziger, B. (2007): Das BAG und das Arbeitszeitgesetz - Aktuelle Tendenzen, in: Der Betrieb (DB) 60 (24), S. 1356-1358 